\title{
On a unified integral operator for $\varphi$-convex functions
}

\author{
Young Chel Kwun', Moquddsa Zahra ${ }^{2 *}$, Ghulam Farid ${ }^{3}$, Saira Zainab² and Shin Min Kang ${ }^{4}$
}

${ }^{*}$ Correspondence:

moquddsazahra@gmail.com

${ }^{2}$ Department of Mathematics, University of Wah, Wah Cantt, Pakistan

Full list of author information is available at the end of the article

\begin{abstract}
Integral operators have a very vital role in diverse fields of science and engineering. In this paper, we use $\varphi$-convex functions for unified integral operators to obtain their upper bounds and upper and lower bounds for symmetric $\varphi$-convex functions in the form of a Hadamard inequality. Also, for $\varphi$-convex functions, we obtain bounds of different known fractional and conformable fractional integrals. The results of this paper are applicable to convex functions.
\end{abstract}

Keywords: Convex function; $\varphi$-convex function; Integral operators; Fractional integrals; Conformable fractional integrals; Bounds

\section{Introduction and preliminaries}

Some very interesting properties of convex functions make them important in mathematical analysis. It should be noted that in new problems related to convexity, generalized assumptions about convex functions are necessary to obtain applicable results. During the recent era, there have been several attempts to generalize the notion of convex functions. Many important generalizations can be found for convex functions, such as $\alpha$-convex, $m$-convex, $h$-convex, $(\alpha, m)$-convex, $(h, m)$-convex, $s$-convex, $(s, m)$-convex, GA-convex, GG-convex, and preinvex functions $[1,3,5,9,11,13,14,17,18,20,24]$.

Definition 1 ([21]) A function $f: I \longrightarrow \mathbb{R}$ is said to be convex if

$$
f(t x+(1-t) y) \leq t f(x)+(1-t) f(y)
$$

for all $x, y \in I$ and $t \in[0,1]$, where $I \subseteq \mathbb{R}$ is an interval. If inequality (1.1) is reversed, then $f$ is called a concave function.

Definition 2 ([7]) A function $f: I \longrightarrow \mathbb{R}$ is said to be $\varphi$-convex if

$$
f(t x+(1-t) y) \leq f(y)+t \varphi(f(x), f(y))
$$

for all $x, y \in I$ and $t \in[0,1]$, where $I \subseteq \mathbb{R}$ is a convex set, and $\varphi: f(I) \times f(I) \longrightarrow \mathbb{R}$ is a bifunction.

(c) The Author(s) 2020. This article is licensed under a Creative Commons Attribution 4.0 International License, which permits use, sharing, adaptation, distribution and reproduction in any medium or format, as long as you give appropriate credit to the original author(s) and the source, provide a link to the Creative Commons licence, and indicate if changes were made. The images or other third party material in this article are included in the article's Creative Commons licence, unless indicated otherwise in a credit line to the material. If material is not included in the article's Creative Commons licence and your intended use is not permitted by statutory regulation or exceeds the permitted use, you will need to obtain permission directly from the copyright holder. To view a copy of this licence, visit http://creativecommons.org/licenses/by/4.0/. 
For $\varphi(x, y)=x-y$, the $\varphi$-convex functions reduce to the convex functions. Note that every convex function is $\varphi$-convex, but the converse is not true.

Example 1 ([7]) The function

$$
f(x)= \begin{cases}-x, & x \geq 0 \\ x, & x<0\end{cases}
$$

where $\varphi:(-\infty, 0] \times(-\infty, 0] \rightarrow \mathbb{R}$ is defined by

$$
\varphi(x, y)= \begin{cases}x, & y=0, \\ -y, & x=0, \\ -x-y, & x<0, y<0,\end{cases}
$$

is $\varphi$-convex but not a convex function.

Integral operators play a very vital role in the study of fractional derivatives and fractional integrals. Next, we give definitions of some integral operators, which will be utilized in the results of this paper.

Definition 3 ([15]) Let $f \in L\left[x_{0}, y_{0}\right]$, and let $g$ be a positive increasing function on $\left(x_{0}, y_{0}\right]$ with continuous derivative on $\left(x_{0}, y_{0}\right)$. The left- and right-sided fractional integral operators of $f$ with respect to $g$ on $\left[x_{0}, y_{0}\right]$ of order $\mu$, where $\Re(\mu)>0$, are given by

$$
\begin{aligned}
& { }_{g}^{\mu} I_{x_{0}^{+}} f(x)=\frac{1}{\Gamma(\mu)} \int_{x_{0}}^{x}(g(x)-g(t))^{\mu-1} g^{\prime}(t) f(t) d t, \quad x>x_{0}, \\
& { }_{g}^{\mu} I_{y_{0}} f(x)=\frac{1}{\Gamma(\mu)} \int_{x}^{y_{0}}(g(t)-g(x))^{\mu-1} g^{\prime}(t) f(t) d t, \quad x<y_{0},
\end{aligned}
$$

where $\Gamma$ is the gamma function.

Definition 4 ([16]) Let $f \in L\left[x_{0}, y_{0}\right]$, and let $g$ be positive increasing function on $\left(x_{0}, y_{0}\right]$ with continuous derivative on $\left(x_{0}, y_{0}\right)$. The left- and right-sided $k$-fractional integral operators of $f$ with respect to $g$ on $\left[x_{0}, y_{0}\right]$ of order $\mu$, where $\Re(\mu), k>0$, are given by

$$
\begin{aligned}
& { }_{g}^{\mu} I_{x_{0}^{+}}^{k} f(x)=\frac{1}{k \Gamma_{k}(\mu)} \int_{x_{0}}^{x}(g(x)-g(t))^{\frac{\mu}{k}-1} g^{\prime}(t) f(t) d t, \quad x>x_{0}, \\
& { }_{g}^{\mu} I_{y_{0}^{-}}^{k} f(x)=\frac{1}{k \Gamma_{k}(\mu)} \int_{x}^{y_{0}}(g(t)-g(x))^{\frac{\mu}{k}-1} g^{\prime}(t) f(t) d t, \quad x<y_{0},
\end{aligned}
$$

where $\Gamma_{k}$ is the $k$-gamma function.

Definition 5 ([2]) Let $f \in L_{1}\left[x_{0}, y_{0}\right]$ and $x \in\left[x_{0}, y_{0}\right]$. Also, let $\eta, \mu, \alpha, \xi, \gamma, \zeta \in C, \Re(\mu), \Re(\alpha)$, $\Re(\xi)>0, \Re(\zeta)>\Re(\gamma)>0$ with $p \geq 0, \delta>0$, and $0<k \leq \delta+\Re(\mu)$. Then the left- and rightsided generalized fractional integral operators $\epsilon_{\mu, \alpha, \xi, \eta, x_{0}^{*}}^{\gamma, \delta, k, \zeta} f$ and $\epsilon_{\mu, \alpha, \xi, \eta, \eta, y_{0}^{-}}^{\gamma, \delta, k, \zeta} f$ are defined by

$$
\left(\epsilon_{\mu, \alpha, \xi, \eta, x_{0}^{+}}^{\gamma, \delta, k, \zeta} f\right)(x ; p)=\int_{x_{0}}^{x}(x-t)^{\alpha-1} E_{\mu, \alpha, \xi}^{\gamma, \delta, k, \zeta}\left(\eta(x-t)^{\mu} ; p\right) f(t) d t
$$




$$
\left(\epsilon_{\mu, \alpha, \xi, \eta, y_{0}^{-}}^{\gamma, \delta, k, \zeta} f\right)(x ; p)=\int_{x}^{y_{0}}(t-x)^{\alpha-1} E_{\mu, \alpha, \xi}^{\gamma, \delta, k, \zeta}\left(\eta(t-x)^{\mu} ; p\right) f(t) d t
$$

where $E_{\mu, \alpha, \xi}^{\gamma, \delta, k, \zeta}(t ; p)$ is the extended generalized Mittag-Leffler function defined as

$$
E_{\mu, \alpha, \xi}^{\gamma, \delta, k, \xi}(t ; p)=\sum_{n=0}^{\infty} \frac{\beta_{p}(\gamma+n k, \zeta-\gamma)}{\beta(\gamma, \zeta-\gamma)} \frac{(\zeta)_{n k}}{\Gamma(\mu n+\alpha)} \frac{t^{n}}{(\xi)_{n \delta}}
$$

Farid [6] (see also [16]) defined unified integral operators and proved that these integral operators are bounded, linear, and thus continuous.

Definition $6([6])$ Let $f, g:\left[x_{0}, y_{0}\right] \rightarrow \mathbb{R}$, where $0<x_{0}<y_{0}$, be functions such that $\mathrm{f}$ is positive and integrable over $\left[x_{0}, y_{0}\right]$ and $g$ is differentiable and strictly increasing. Also, let $\frac{\Psi}{x}$ be an increasing function on $\left[x_{0}, \infty\right)$, and let $\alpha, \xi, \gamma, \zeta \in \mathbb{C}, p, \mu, \delta \geq 0$, and $0<k \leq \delta+\mu$. Then for $x \in\left[x_{0}, y_{0}\right]$, the left and right integral operators are given by

$$
\begin{aligned}
& \left({ }_{g} F_{\mu, \alpha, \xi, x_{0}^{+}}^{\Psi, \gamma, \delta, k} f\right)(x ; p)=\int_{x_{0}}^{x} G_{x}^{y}\left(E_{\mu, \alpha, \xi}^{\gamma, \delta, k, \zeta}, g ; \Psi\right) g^{\prime}(y) f(y) d y \\
& \left({ }_{g} F_{\mu, \alpha, \xi, y_{0}^{-}}^{\Psi, \gamma, k, \zeta} f\right)(x ; p)=\int_{x}^{y_{0}} G_{y}^{x}\left(E_{\mu, \alpha, \xi}^{\gamma, \delta, k, \zeta}, g ; \Psi\right) g^{\prime}(y) f(y) d y
\end{aligned}
$$

where

$$
G_{x}^{y}\left(E_{\mu, \alpha, \xi}^{\gamma, \delta, k, \zeta}, g ; \Psi\right)=\frac{\Psi(g(x)-g(y))}{g(x)-g(y)} E_{\mu, \alpha, \xi}^{\gamma, \delta, k, \zeta}\left(\eta(g(x)-g(y))^{\mu} ; p\right) .
$$

For the particular choice of $\Psi, g$ and the parameters involved in Mittag-Leffler functions, several conformable and fractional integrals can be obtained; see [16, Remarks 6 and 7]. In [16], some bounds of the above operators have been proved for convex functions.

Theorem 1 Let $f:\left[x_{0}, y_{0}\right] \longrightarrow \mathbb{R}$ be a positive convex function for $0<x_{0}<y_{0}$, and let $g$ : $\left[x_{0}, y_{0}\right] \longrightarrow \mathbb{R}$ be differentiable and strictly increasing. Also, let $\frac{\Psi}{x}$ be an increasing function on $\left[x_{0}, y_{0}\right]$, and let $\eta, \alpha, \xi, \gamma, \zeta \in \mathbb{C}, p, \mu \geq 0, \delta \geq 0$, and $0<k \leq \delta+\mu$. Then we have the following bound for $x \in\left[x_{0}, y_{0}\right]$ :

$$
\begin{aligned}
& \left({ }_{g} F_{\mu, \alpha, \xi, x_{0}^{+}}^{\Psi, \gamma, \delta, k} f\right)(x ; p)+\left({ }_{g} F_{\mu, \alpha, \xi, y_{0}^{-}}^{\Psi, \gamma, k, \zeta} f\right)(x ; p) \\
& \leq E_{\mu, \alpha, \xi}^{\gamma, \delta, k, \zeta}\left(\eta\left(g(x)-g\left(x_{0}\right)\right)^{\mu} ; p\right) \\
& \quad \times \Psi\left(g(x)-g\left(x_{0}\right)\right)\left(f(x)+f\left(x_{0}\right)\right)+E_{\mu, \alpha, \xi}^{\gamma, \delta, k, \zeta}\left(\eta\left(g\left(y_{0}\right)-g(x)\right)^{\mu} ; p\right) \Psi\left(g\left(y_{0}\right)-g(x)\right) \\
& \quad \times\left(f(x)+f\left(y_{0}\right)\right) .
\end{aligned}
$$

Further, the following bounds hold as a version of the Hadamard inequality.

Theorem 2 Along with assumptions of the Theorem 1, iff is symmetric about $\frac{x_{0}+y_{0}}{2}$, then we have the following inequalities:

$$
f\left(\frac{x_{0}+y_{0}}{2}\right)\left(\left({ }_{g} F_{\mu, \alpha, \xi, y_{0}^{-}}^{\Psi, \gamma, \delta, \zeta} 1\right)\left(x_{0} ; p\right)+\left({ }_{g} F_{\mu, \alpha, \xi, x_{0}^{+}}^{\Psi, \gamma, k, k} 1\right)\left(y_{0} ; p\right)\right)
$$




$$
\begin{aligned}
& \leq\left({ }_{g} F_{\mu, \alpha, \xi, y}^{\Psi, \gamma, \delta, k, \zeta} f\right)\left(x_{0} ; p\right)+\left({ }_{g} F_{\mu, \alpha, \xi, x_{0}^{+}}^{\Psi, \gamma, \delta, k} f\right)\left(y_{0} ; p\right) \\
& \leq 2 \Psi\left(g\left(y_{0}\right)-g\left(x_{0}\right)\right) E_{\mu, \alpha, \xi, \zeta}^{\gamma, \delta, k, \zeta}\left(\eta\left(g\left(y_{0}\right)-g\left(x_{0}\right)\right)^{\mu} ; p\right)\left(f\left(x_{0}\right)+f\left(y_{0}\right)\right) .
\end{aligned}
$$

Moreover, the following result is produced by defining unified operators for the convolution $f * g$ of functions $f$ and $g$.

Theorem 3 Let $f, g:\left[x_{0}, y_{0}\right] \longrightarrow \mathbb{R}$ be a differentiable functions such that $\left|f^{\prime}\right|$ is convex, $0<x_{0}<y_{0}$, and $g$ is a strictly increasing function. Also, let $\frac{\Psi}{x}$ be an increasing function, and let $\eta, \alpha, \xi, \gamma, \zeta \in \mathbb{C}, p, \mu, \delta \geq 0$, and $0<k \leq \delta+\mu$. Then we have the following modulus inequality for $x \in\left(x_{0}, y_{0}\right)$ :

$$
\begin{aligned}
& \left|\left({ }_{g} F_{\mu, \alpha, \xi, x_{0}^{+}}^{\Psi, \gamma, \delta, k,} f * g\right)(x ; p)+\left({ }_{g} F_{\mu, \alpha, \xi, y, y_{0}^{-}}^{\Psi, \gamma, \delta, k} f * g\right)(x ; p)\right| \\
& \leq E_{\mu, \alpha, \xi}^{\gamma, \delta, k, \zeta}\left(\eta\left(g(x)-g\left(x_{0}\right)\right)^{\mu} ; p\right) \Psi\left(g(x)-g\left(x_{0}\right)\right)\left(\left|f^{\prime}(x)\right|+\left|f^{\prime}\left(x_{0}\right)\right|\right) \\
& \quad+E_{\mu, \alpha, \xi}^{\gamma, \delta, k, \zeta}\left(\eta\left(g\left(y_{0}\right)-g(x)\right)^{\mu} ; p\right) \Psi\left(g\left(y_{0}\right)-g(x)\right)\left(\left|f^{\prime}(x)\right|+\left|f^{\prime}\left(y_{0}\right)\right|\right),
\end{aligned}
$$

where

$$
\begin{aligned}
& \left({ }_{g} F_{\mu, \alpha, \xi, \xi, x_{0}^{+}}^{\Psi, \gamma, \delta, k} f * g\right)(x ; p)=\int_{x_{0}}^{x} G_{x}^{t}\left(E_{\mu, \alpha, \xi}^{\gamma, \delta, k, \zeta}, g ; \Psi\right) f^{\prime}(t) d(g(t)), \\
& \left({ }_{g} F_{\mu, \alpha, \xi, y, y_{0}^{-}}^{\Psi, \gamma, \zeta, \zeta} f * g\right)(x ; p)=\int_{x}^{y_{0}} G_{t}^{x}\left(E_{\mu, \alpha, \xi}^{\gamma, \delta, k, \zeta}, g ; \Psi\right) f^{\prime}(t) d(g(t)) .
\end{aligned}
$$

In Sect. 2, we use $\varphi$-convex functions to obtain bounds of integral operators given in Definition 6. Moreover, we achieve Hadamard-type bounds using the additional condition of symmetry. Also, we get some particular bounds by the $\varphi$-convexity of $\left|f^{\prime}\right|$ and defining a convenient integral operator of convolution of two functions. In Sect. 3, we give some applications of the presented results.

\section{Main results}

Throughout this section, we assume that

$$
I\left(x_{0}, y_{0} ; g\right):=\frac{1}{y_{0}-x_{0}} \int_{x_{0}}^{y_{0}} g(t) d t
$$

Theorem 4 Let $f:\left[x_{0}, y_{0}\right] \longrightarrow \mathbb{R}$ be a positive $\varphi$-convex function, and let $g:\left[x_{0}, y_{0}\right] \longrightarrow$ $\mathbb{R}$ be a differentiable strictly increasing function. Also, let $\frac{\Psi}{x}$ be an increasing function on $\left[x_{0}, y_{0}\right]$, and let $\eta, \alpha, \xi, \gamma, \zeta \in \mathbb{C}, p, \mu, \nu, \delta \geq 0,0<k \leq \delta+\mu$, and $0<k \leq \delta+v$. Then for $x \in\left[x_{0}, y_{0}\right]$, we have

$$
\begin{aligned}
& \left({ }_{g} F_{\mu, \alpha, \xi, x_{0}^{+}}^{\Psi, \gamma, \delta, k} f\right)(x ; p) \\
& \leq E_{\mu, \alpha, \xi}^{\gamma, \delta, k, \zeta}\left(\eta\left(g(x)-g\left(x_{0}\right)\right)^{\mu} ; p\right) \Psi\left(g(x)-g\left(x_{0}\right)\right) f(x) \\
& \quad+G_{x}^{x_{0}}\left(E_{\mu, \alpha, \xi}^{\gamma, \delta, k, \zeta}, g ; \Psi\right) \varphi\left(f\left(x_{0}\right), f(x)\right)\left(I\left(x_{0}, x, g\right)-g\left(x_{0}\right)\right), \\
& \left({ }_{g} F_{v, \alpha, \xi, \xi, y_{0}^{-}}^{\Psi, \gamma} f\right)(x ; p) \leq E_{v, \alpha, \xi}^{\gamma, \delta, k, \zeta}\left(\eta\left(g\left(y_{0}\right)-g(x)\right)^{\nu} ; p\right) \Psi\left(g\left(y_{0}\right)-g(x)\right) f\left(y_{0}\right) \\
& +G_{y_{0}}^{x}\left(E_{v, \alpha, \xi}^{\gamma, \delta, k, \zeta}, g ; \Psi\right) \varphi\left(f(x), f\left(y_{0}\right)\right)\left(I\left(x, y_{0} ; g\right)-g(x)\right),
\end{aligned}
$$




$$
\begin{aligned}
\left({ }_{g} F_{\mu, \alpha, \xi, x_{0}^{+}}^{\Psi, \gamma, \delta, k} f\right)(x ; p)+\left({ }_{g} F_{v, \alpha, \xi, y_{0}^{\prime}}^{\Psi, \gamma, \delta, \zeta} f\right)(x ; p) \\
\leq \\
\quad E_{\mu, \alpha, \xi}^{\gamma, \delta, k, \zeta}\left(\eta\left(g(x)-g\left(x_{0}\right)\right)^{\mu} ; p\right) \Psi\left(g(x)-g\left(x_{0}\right)\right) f(x) \\
\quad+G_{x}^{x_{0}}\left(E_{\mu, \alpha, \xi}^{\gamma, \delta, k, \zeta}, g ; \Psi\right) \varphi\left(f\left(x_{0}\right), f(x)\right)\left(I\left(x_{0}, x ; g\right)-g\left(x_{0}\right)\right) \\
\quad+E_{v, \alpha, \xi}^{\gamma, \delta, k, \zeta}\left(\eta\left(g\left(y_{0}\right)-g(x)\right)^{\nu} ; p\right) \Psi\left(g\left(y_{0}\right)-g(x)\right) f\left(y_{0}\right) \\
\quad+G_{y_{0}}^{x}\left(E_{v, \alpha, \xi}^{\gamma, \delta, k, \zeta}, g ; \Psi\right) \varphi\left(f(x), f\left(y_{0}\right)\right)\left(I\left(x, y_{0} ; g\right)-g(x)\right) .
\end{aligned}
$$

Proof We have the following inequality for the kernel defined in (1.14) and an increasing function $g$ :

$$
G_{x}^{t}\left(E_{\mu, \alpha, \xi}^{\gamma, \delta, k, \zeta}, g ; \Psi\right) g^{\prime}(t) \leq G_{x}^{x_{0}}\left(E_{\mu, \alpha, \xi}^{\gamma, \delta, k, \zeta}, g ; \Psi\right) g^{\prime}(t), \quad t \in\left[x_{0}, x\right], x \in\left(x_{0}, y_{0}\right) .
$$

Using the $\varphi$-convexity of $f$, we have

$$
f(t) \leq f(x)+\frac{x-t}{x-x_{0}} \varphi\left(f\left(x_{0}\right), f(x)\right) .
$$

Inequalities (2.4) and (2.5) constitute the following integral inequality:

$$
\begin{aligned}
& \int_{x_{0}}^{x} G_{x}^{t}\left(E_{\mu, \alpha, \xi}^{\gamma, \delta, k, \zeta}, g ; \Psi\right) g^{\prime}(t) f(t) d t \\
& \quad \leq f(x) G_{x}^{x_{0}}\left(E_{\mu, \alpha, \xi, \xi, \xi, \zeta}^{\gamma, \xi} \Psi\right) \int_{x_{0}}^{x} g^{\prime}(t) d t \\
& \quad+\frac{\varphi\left(f\left(x_{0}\right), f(x)\right)}{x-x_{0}} G_{x}^{x_{0}}\left(E_{\mu, \alpha, \xi}^{\gamma, \delta, k, \zeta}, g ; \Psi\right) \int_{x_{0}}^{x}(x-t) g^{\prime}(t) d t .
\end{aligned}
$$

Using (1.12) of Definition 6 on the left-hand side of inequality (2.6) and integrating the right-hand side, we get

$$
\begin{aligned}
& \left({ }_{g} F_{\mu, \alpha, \xi, x_{0}^{+}}^{\Psi, \gamma, \delta, k, \zeta} f\right)(x ; p) \\
& \quad \leq E_{\mu, \alpha, \xi}^{\gamma, \delta, k, \zeta}\left(\eta\left(g(x)-g\left(x_{0}\right)\right)^{\mu} ; p\right) \Psi\left(g(x)-g\left(x_{0}\right) f(x)\right. \\
& \quad+G_{x}^{x_{0}}\left(E_{\mu, \alpha, \xi}^{\gamma, \delta, k, \zeta}, g ; \Psi\right) \varphi\left(f\left(x_{0}\right), f(x)\right)\left(I\left(x_{0}, x ; g\right)-g\left(x_{0}\right)\right) .
\end{aligned}
$$

Now using the same technique for $t \in\left(x, y_{0}\right]$ and $x \in\left(x_{0}, y_{0}\right)$, we can write

$$
G_{t}^{x}\left(E_{v, \alpha, \xi}^{\gamma, \delta, k, \zeta}, g ; \Psi\right) g^{\prime}(t) \leq G_{y_{0}}^{x}\left(E_{v, \alpha, \xi}^{\gamma, \delta, k, \zeta}, g ; \Psi\right) g^{\prime}(t)
$$

Using the $\varphi$-convexity of $f$, we have

$$
f(t) \leq f\left(y_{0}\right)+\frac{y_{0}-t}{y_{0}-x} \varphi\left(f(x), f\left(y_{0}\right)\right) .
$$

Inequalities (2.8) and (2.9) constitute the following inequality:

$$
\int_{x}^{y_{0}} G_{t}^{x}\left(E_{v, \alpha, \xi}^{\gamma, \delta, k, \zeta}, g ; \Psi\right) g^{\prime}(t) f(t) d t
$$




$$
\leq G_{y_{0}}^{x}\left(E_{v, \alpha, \xi}^{\gamma, \delta, k, \zeta}, g ; \Psi\right)\left(f\left(y_{0}\right) \int_{x}^{y_{0}} g^{\prime}(t) d t+\frac{\varphi\left(f(x), f\left(y_{0}\right)\right)}{y_{0}-x} \int_{x}^{y_{0}}\left(y_{0}-t\right) g^{\prime}(t) d t\right) .
$$

Using (1.13) of Definition 6 on the left-hand side and integrating by parts the right-hand side, we get

$$
\begin{aligned}
\left({ }_{g} F_{\nu, \alpha, \xi, y_{0}^{-}}^{\Psi, \gamma, \delta, k} f\right)(x ; p) \leq & E_{\nu, \alpha, \xi}^{\gamma, \delta, k, \zeta}\left(\eta\left(g\left(y_{0}\right)-g(x)\right)^{\nu} ; p\right) \Psi\left(g\left(y_{0}\right)-g(x)\right) f\left(y_{0}\right) \\
& +G_{y_{0}}^{x}\left(E_{\nu, \alpha, \xi}^{\gamma, \delta, k, \zeta}, g ; \Psi\right) \varphi\left(f(x), f\left(y_{0}\right)\right)\left(I\left(x, y_{0} ; g\right)-g(x)\right) .
\end{aligned}
$$

We obtain inequality (2.3) by summing (2.7) and (2.10).

Corollary 1 By setting $\mu=v$ in (2.3) we get

$$
\begin{aligned}
\left({ }_{g} F_{\mu, \alpha, \xi, x_{0}^{+}}^{\Psi, \gamma, \delta, k, \zeta} f\right)(x ; p)+\left({ }_{g} F_{\mu, \alpha, \xi, y,}^{\Psi, \gamma, \delta, k}\right. & f)(x ; p) \\
\leq & E_{\mu, \alpha, \xi}^{\gamma, \delta, k, \zeta}\left(\eta\left(g(x)-g\left(x_{0}\right)\right)^{\mu} ; p\right) \Psi\left(g(x)-g\left(x_{0}\right)\right) f(x) \\
& +G_{x}^{x_{0}}\left(E_{\mu, \alpha, \xi}^{\gamma, \delta, k, \zeta}, g ; \Psi\right) \varphi\left(f\left(x_{0}\right), f(x)\right)\left(I\left(x_{0}, x ; g\right)-g\left(x_{0}\right)\right) \\
& +E_{\mu, \alpha, \xi}^{\gamma, \delta, k, \zeta}\left(\eta\left(g\left(y_{0}\right)-g(x)\right)^{\mu} ; p\right) \Psi\left(g\left(y_{0}\right)-g(x)\right) f\left(y_{0}\right) \\
& +G_{y_{0}}^{x}\left(E_{\mu, \alpha, \xi, \xi}^{\gamma, \delta, \zeta,}, g ; \Psi\right) \varphi\left(f(x), f\left(y_{0}\right)\right)\left(I\left(x, y_{0} ; g\right)-g(x)\right) .
\end{aligned}
$$

Remark 1 If we take $\varphi(x, y)=x-y$ in (2.11), we get inequality (1.15) of Theorem 1 .

We will need the following lemma in proving the upcoming result.

Lemma 1 Let $f:\left[x_{0}, y_{0}\right] \rightarrow \mathbb{R}$ be a $\varphi$-convex function. If $f(x)=f\left(x_{0}+y_{0}-x\right), x \in\left[x_{0}, y_{0}\right]$, then we have the following inequality:

$$
f\left(\frac{x_{0}+y_{0}}{2}\right) \leq f(x)+\frac{1}{2} \varphi(f(x), f(x)) \text {. }
$$

Proof Since $f$ is $\varphi$-convex, we get

$$
\begin{aligned}
f\left(\frac{x_{0}+y_{0}}{2}\right) \leq & f\left(\frac{x-x_{0}}{y_{0}-x_{0}} x_{0}+\frac{y_{0}-x}{y_{0}-x_{0}} y_{0}\right) \\
& +\frac{1}{2} \varphi\left(f\left(\frac{x-x_{0}}{y_{0}-x_{0}} y_{0}+\frac{y_{0}-x}{y_{0}-x_{0}} x_{0}\right), f\left(\frac{x-x_{0}}{y_{0}-x_{0}} x_{0}+\frac{y_{0}-x}{y_{0}-x_{0}} y_{0}\right)\right) \\
\leq & f\left(x_{0}+y_{0}-x\right)+\frac{1}{2} \varphi\left(f(x), f\left(x_{0}+y_{0}-x\right)\right) .
\end{aligned}
$$

Using $f\left(x_{0}+y_{0}-x\right)=f(x)$ in this inequality, we get inequality (2.12).

Remark 2 For $\varphi(x, y)=x-y$, Lemma 1 reduces to [16, Lemma 1].

Theorem 5 Along with the assumptions of Theorem 4, if $f\left(x_{0}+y_{0}-x\right)=f(x)$ and $\varphi(x, y)=$ $x+y$, then

$$
\frac{1}{2} f\left(\frac{x_{0}+y_{0}}{2}\right)\left(\left({ }_{g} F_{\mu, \alpha, \xi, y, y_{0}}^{\Psi, \gamma, \delta, k, \zeta} 1\right)\left(x_{0} ; p\right)+\left({ }_{g} F_{\mu, \alpha, \xi, x_{0}^{+}}^{\Psi, \gamma, \delta, k, \zeta} 1\right)\left(y_{0} ; p\right)\right)
$$




$$
\begin{aligned}
\leq & \left({ }_{g} F_{\mu, \alpha, \xi, y_{0}^{-}}^{\Psi, \gamma, \delta, k, \zeta} f\right)\left(x_{0} ; p\right)+\left({ }_{g} F_{\mu, \alpha, \xi, x_{0}^{+}}^{\Psi, \gamma, \delta, k, \zeta} f\right)\left(y_{0} ; p\right) \\
\leq & 2 \Psi\left(g\left(y_{0}\right)-g\left(x_{0}\right)\right) E_{\mu, \alpha, \xi}^{\gamma, \delta, k, \zeta}\left(\eta\left(g\left(y_{0}\right)-g\left(x_{0}\right)\right)^{\mu} ; p\right) f\left(y_{0}\right) \\
& +2\left(f\left(x_{0}\right)+f\left(y_{0}\right)\right) G_{y_{0}}^{x_{0}}\left(E_{\mu, \alpha, \xi}^{\gamma, \delta, k, \zeta}, g ; \Psi\right)\left(I\left(x_{0}, y_{0} ; g\right)-g\left(x_{0}\right)\right) .
\end{aligned}
$$

Proof We have the following inequality for the kernel defined in (1.14) and an increasing function $g$ :

$$
G_{x}^{x_{0}}\left(E_{\mu, \alpha, \xi}^{\gamma, \delta, k, \zeta}, g ; \Psi\right) g^{\prime}(x) \leq G_{y_{0}}^{x_{0}}\left(E_{\mu, \alpha, \xi}^{\gamma, \delta, k, \zeta}, g ; \Psi\right) g^{\prime}(x), \quad x \in\left(x_{0}, y_{0}\right) .
$$

Using the $\varphi$-convexity of $f$ over $\left[x_{0}, y_{0}\right]$, we have

$$
f(x) \leq f\left(y_{0}\right)+\frac{y_{0}-x}{y_{0}-x_{0}} \varphi\left(f\left(x_{0}\right), f\left(y_{0}\right)\right) .
$$

Inequalities (2.14) and (2.15) constitute the following integral inequality:

$$
\begin{aligned}
& \int_{x_{0}}^{y_{0}} G_{x}^{x_{0}}\left(E_{\mu, \alpha, \xi}^{\gamma, \delta, k, \zeta}, g ; \Psi\right) f(x) g^{\prime}(x) d x \\
& \leq f\left(y_{0}\right) G_{y_{0}}^{x_{0}}\left(E_{\mu, \alpha, \xi}^{\gamma, \delta, k, \zeta}, g ; \Psi\right) \int_{x_{0}}^{y_{0}} g^{\prime}(x) d x \\
& \quad+\frac{\varphi\left(f\left(x_{0}\right), f\left(y_{0}\right)\right)}{y_{0}-x_{0}} G_{y_{0}}^{x_{0}}\left(E_{\mu, \alpha, \xi}^{\gamma, \delta, k, \zeta}, g ; \Psi\right) \int_{x_{0}}^{y_{0}}\left(y_{0}-x\right) g^{\prime}(x) d x
\end{aligned}
$$

Using (1.13) of Definition 6 on left-hand side, integrating the right-hand side, and using $\varphi(x, y)=x+y$, this inequality gives

$$
\begin{aligned}
\left({ }_{g} F_{\mu, \alpha, \xi, y}^{\Psi, \gamma, \delta, k, \zeta} f\right)\left(x_{0} ; p\right) \\
\quad \leq E_{\mu, \alpha, \xi}^{\gamma, \delta, k, \zeta}\left(\eta\left(g\left(y_{0}\right)-g\left(x_{0}\right)\right)^{\mu} ; p\right) \Psi\left(g\left(y_{0}\right)-g\left(x_{0}\right)\right) f\left(y_{0}\right) \\
\quad+G_{y_{0}}^{x_{0}}\left(E_{\mu, \alpha, \xi}^{\gamma, \delta, k, \zeta}, g ; \Psi\right)\left(f\left(x_{0}\right)+f\left(y_{0}\right)\right)\left(I\left(x_{0}, y_{0} ; g\right)-g\left(x_{0}\right)\right) .
\end{aligned}
$$

Also, we have the following inequality:

$$
G_{y_{0}}^{x}\left(E_{\mu, \alpha, \xi}^{\gamma, \delta, k, \zeta}, g ; \Psi\right) g^{\prime}(x) \leq G_{y_{0}}^{x_{0}}\left(E_{\mu, \alpha, \xi}^{\gamma, \delta, k, \zeta}, g ; \Psi\right) g^{\prime}(x), \quad x \in\left(x_{0}, y_{0}\right) \text {. }
$$

Inequalities (2.15) and (2.17) constitute the following integral inequality:

$$
\begin{aligned}
\int_{x_{0}}^{y_{0}} & G_{y_{0}}^{x}\left(E_{\mu, \alpha, \xi}^{\gamma, \delta, k, \zeta}, g ; \Psi\right) g^{\prime}(x) f(x) d x \\
\leq & G_{y_{0}}^{x_{0}}\left(E_{\mu, \alpha, \xi}^{\gamma, \delta, k, \zeta}, g ; \Psi\right) \\
& \times\left(f\left(y_{0}\right) \int_{x_{0}}^{y_{0}} g^{\prime}(x) d x+\frac{\varphi\left(f\left(x_{0}\right), f\left(y_{0}\right)\right)}{y_{0}-x_{0}} \int_{x_{0}}^{y_{0}} g^{\prime}(x)\left(y_{0}-x\right) d x\right) .
\end{aligned}
$$

Using (1.12) of Definition 6 on the left-hand side, integrating the right-hand side, and using $\varphi(x, y)=x+y$, this inequality gives

$$
\left({ }_{g} F_{\mu, \alpha, \xi, x_{0}^{+}}^{\Psi, \gamma, \delta} f\right)\left(y_{0} ; p\right)
$$




$$
\begin{aligned}
\leq & E_{\mu, \alpha, \xi}^{\gamma, \delta, k, \zeta}\left(\eta\left(g\left(y_{0}\right)-g\left(x_{0}\right)\right)^{\mu} ; p\right) \Psi\left(g\left(y_{0}\right)-g\left(x_{0}\right)\right) f\left(y_{0}\right) \\
& +G_{y_{0}}^{x_{0}}\left(E_{\mu, \alpha, \xi}^{\gamma, \delta, k, \zeta}, g ; \Psi\right)\left(f\left(x_{0}\right)+f\left(y_{0}\right)\right)\left(I\left(x_{0}, y_{0} ; g\right)-g\left(x_{0}\right)\right) .
\end{aligned}
$$

Now from (2.12) of Lemma 1 we can write

$$
\begin{aligned}
& \int_{x_{0}}^{y_{0}} f\left(\frac{x_{0}+y_{0}}{2}\right) G_{x}^{x_{0}}\left(E_{\mu, \alpha, \xi}^{\gamma, \delta, k, \zeta}, g ; \Psi\right) g^{\prime}(x) d x \\
& \leq \int_{x_{0}}^{y_{0}} G_{x}^{x_{0}}\left(E_{\mu, \alpha, \xi}^{\gamma, \delta, k, \zeta}, g ; \Psi\right) g^{\prime}(x) f(x) d x \\
& \quad+\frac{1}{2} \int_{x_{0}}^{y_{0}} G_{x}^{x_{0}}\left(E_{\mu, \alpha, \xi}^{\gamma, \delta, k, \zeta} g ; \Psi\right) g^{\prime}(x) \varphi(f(x), f(x)) d x
\end{aligned}
$$

Using (1.13) of Definition 6 and $\varphi(x, y)=x+y$, we get

$$
f\left(\frac{x_{0}+y_{0}}{2}\right)\left(F_{g} F_{\mu, \alpha, \xi, \gamma, y_{0}^{-}}^{\Psi, \gamma, k} 1\right)\left(x_{0} ; p\right) \leq 2\left(F_{g}^{\Psi, \alpha, \alpha, \xi, y_{0}^{-}} f\right)\left(x_{0} ; p\right)
$$

Again using (2.12) of Lemma 1, we can write

$$
\begin{aligned}
& \int_{x_{0}}^{y_{0}} f\left(\frac{x_{0}+y_{0}}{2}\right) G_{y_{0}}^{x}\left(E_{\mu, \alpha, \xi}^{\gamma, \delta, k, \zeta}, g ; \Psi\right) g^{\prime}(x) d x \\
& \leq \int_{x_{0}}^{y_{0}} G_{y_{0}}^{x}\left(E_{\mu, \alpha, \xi}^{\gamma, \delta, k, \zeta}, g ; \Psi\right) g^{\prime}(x) f(x) d x \\
& \quad+\frac{1}{2} \int_{x_{0}}^{y_{0}} G_{y_{0}}^{x}\left(E_{\mu, \alpha, \xi}^{\gamma, \delta, k, \zeta}, g ; \Psi\right) g^{\prime}(x) \varphi(f(x), f(x)) d x .
\end{aligned}
$$

Using (1.12) of Definition 6 and $\varphi(x, y)=x+y$, we get

$$
f\left(\frac{x_{0}+y_{0}}{2}\right)\left({ }_{g} F_{\mu, \alpha, \xi, x_{0}^{+}}^{\Psi, \gamma, \delta, k, \zeta} 1\right)\left(y_{0} ; p\right) \leq 2\left({ }_{g} F_{\mu, \alpha, \xi, x_{0}^{+}}^{\Psi, \gamma, \delta, k, \zeta}\right)\left(y_{0} ; p\right) .
$$

We obtain inequality (2.13) by using (2.16), (2.18), (2.19), and (2.21).

Remark 3 By setting $\varphi(x, y)=x-y$ in Theorem 5 we get (1.16) of Theorem 2 .

Theorem 6 Let $f, g:\left[x_{0}, y_{0}\right] \longrightarrow \mathbb{R}$ be two differentiable functions such that $\left|f^{\prime}\right|$ is $\varphi$-convex and $g$ is strictly increasing for $0<x_{0}<y_{0}$. Also, let $\frac{\Psi}{x}$ be an increasing function on $\left[x_{0}, y_{0}\right]$, and let $\alpha, \xi, \gamma, \zeta \in \mathbb{C}, p, \mu, v, \delta \geq 0,0<k \leq \delta+\mu$, and $0<k \leq \delta+v$. Then for $x \in\left(x_{0}, y_{0}\right)$, we have

$$
\begin{aligned}
& \left|\left(_{g} F_{\mu, \alpha, \xi, x_{0}^{+}}^{\Psi, \gamma, \delta, k, \zeta} f * g\right)(x ; p)+\left({ }_{g} F_{v, \alpha, \xi, y_{0}^{-}}^{\Psi, \gamma, \delta, k, \zeta} f * g\right)(x ; p)\right| \\
& \leq E_{\mu, \alpha, \xi}^{\gamma, \delta, k, \zeta}\left(\eta\left(g(x)-g\left(x_{0}\right)\right)^{\mu} ; p\right) \Psi\left(g(x)-g\left(x_{0}\right)\right)\left|f^{\prime}(x)\right| \\
& \quad+G_{x}^{x_{0}}\left(E_{\mu, \alpha, \xi}^{\gamma, \delta, k, \zeta}, g ; \Psi\right) \varphi\left(\left|f^{\prime}\left(x_{0}\right)\right|,\left|f^{\prime}(x)\right|\right)\left(I\left(x_{0}, x ; g\right)-g\left(x_{0}\right)\right) \\
& \quad+E_{v, \alpha, \xi}^{\gamma, \delta, k, \zeta}\left(\eta\left(g\left(y_{0}\right)-g(x)\right)^{\nu} ; p\right) \Psi\left(g\left(y_{0}\right)-g(x)\right)\left|f^{\prime}\left(y_{0}\right)\right| \\
& \quad+G_{y_{0}}^{x}\left(E_{v, \alpha, \xi}^{\gamma, \delta, k, \zeta}, g ; \Psi\right) \varphi\left(\left|f^{\prime}(x)\right|,\left|f^{\prime}\left(y_{0}\right)\right|\right)\left(I\left(x, y_{0} ; g\right)-g(x)\right),
\end{aligned}
$$

where $\left({ }_{g} F_{\mu, \alpha, \xi, x_{0}^{+}}^{\Psi, \gamma, \delta, k,} f * g\right)(x ; p)$ and $\left({ }_{g} F_{\nu, \alpha, \xi, y_{0}^{-}}^{\Psi, \gamma, \delta, \zeta, \zeta} f g\right)(x ; p)$ are as in (1.18) and (1.19). 
Proof The $\varphi$-convexity of $\left|f^{\prime}\right|$ over $\left[x_{0}, y_{0}\right]$ implies

$$
\left|f^{\prime}(t)\right| \leq\left|f^{\prime}(x)\right|+\frac{x-t}{x-x_{0}} \varphi\left(\left|f^{\prime}\left(x_{0}\right)\right|,\left|f^{\prime}(x)\right|\right), \quad t \in\left[x_{0}, x\right] .
$$

By the property of absolute values we can write

$$
\begin{aligned}
& -\left(\left|f^{\prime}(x)\right|+\frac{x-t}{x-x_{0}} \varphi\left(\left|f^{\prime}\left(x_{0}\right)\right|,\left|f^{\prime}(x)\right|\right)\right) \\
& \quad \leq f^{\prime}(t) \leq\left(\left|f^{\prime}(x)\right|+\frac{x-t}{x-x_{0}} \varphi\left(\left|f^{\prime}\left(x_{0}\right)\right|,\left|f^{\prime}(x)\right|\right)\right) .
\end{aligned}
$$

Inequality (2.4) and the second inequality of (2.24) constitute the following inequality:

$$
\begin{aligned}
& \int_{x_{0}}^{x} G_{x}^{t}\left(E_{\mu, \alpha, \xi}^{\gamma, \delta, k, \zeta}, g ; \Psi\right) g^{\prime}(t) f^{\prime}(t) d t \\
& \leq\left|f^{\prime}(x)\right| G_{x}^{x_{0}}\left(E_{\mu, \alpha, \xi}^{\gamma, \delta, k, \zeta}, g ; \Psi\right) \int_{x_{0}}^{x} g^{\prime}(t) d t \\
& \quad+\frac{\varphi\left(\left|f^{\prime}\left(x_{0}\right)\right|,\left|f^{\prime}(x)\right|\right)}{x-x_{0}} G_{x}^{x_{0}}\left(E_{\mu, \alpha, \xi}^{\gamma, \delta, k, \zeta}, g ; \Psi\right) \int_{x_{0}}^{x}(x-t) g^{\prime}(t) d t,
\end{aligned}
$$

from which we get

$$
\begin{aligned}
& \left({ }_{g} F_{\mu, \alpha, \xi, x_{0}^{+}}^{\Psi, \gamma, \delta, k, \zeta} f * g\right)(x ; p) \\
& \quad \leq E_{\mu, \alpha, \xi}^{\gamma, \delta, k, \zeta}\left(\eta\left(g(x)-g\left(x_{0}\right)\right)^{\mu} ; p\right) \Psi\left(g(x)-g\left(x_{0}\right)\right)\left|f^{\prime}(x)\right| \\
& \quad+G_{x}^{x_{0}}\left(E_{\mu, \alpha, \xi}^{\gamma, \delta, k, \zeta}, g ; \Psi\right) \varphi\left(\left|f^{\prime}\left(x_{0}\right)\right|,\left|f^{\prime}(x)\right|\right)\left(I\left(x_{0}, x ; g\right)-g\left(x_{0}\right)\right) .
\end{aligned}
$$

Further, inequality (2.4) and the first inequality of (2.24) produce the following inequality:

$$
\begin{aligned}
\left({ }_{g} F_{\mu, \alpha, \xi, x_{0}^{+}}^{\Psi, \gamma, \delta, k, \zeta} f * g\right)(x ; p) & \\
\quad \geq & -E_{\mu, \alpha, \xi}^{\gamma, \delta, k, \zeta}\left(\eta\left(g(x)-g\left(x_{0}\right)\right)^{\mu} ; p\right) \Psi\left(g(x)-g\left(x_{0}\right)\right)\left|f^{\prime}(x)\right| \\
\quad & \quad G_{x}^{x_{0}}\left(E_{\mu, \alpha, \xi}^{\gamma, \delta, k, \zeta}, g ; \Psi\right) \varphi\left(\left|f^{\prime}\left(x_{0}\right)\right|,\left|f^{\prime}(x)\right|\right)\left(I\left(x_{0}, x ; g\right)-g\left(x_{0}\right)\right) .
\end{aligned}
$$

Now using the $\varphi$-convexity of $\left|f^{\prime}\right|$ over $\left[x_{0}, y_{0}\right]$, we have

$$
\left|f^{\prime}(t)\right| \leq\left|f^{\prime}\left(y_{0}\right)\right|+\frac{y_{0}-t}{y_{0}-x} \varphi\left(\left|f^{\prime}(x)\right|,\left|f^{\prime}\left(y_{0}\right)\right|\right), \quad t \in\left(x, y_{0}\right]
$$

Also, we have

$$
G_{t}^{x}\left(E_{v, \alpha, \xi}^{\gamma, \delta, k, \zeta}, g ; \Psi\right) g^{\prime}(t) \leq G_{y_{0}}^{x}\left(E_{v, \alpha, \xi}^{\gamma, \delta, k, \zeta}, g ; \Psi\right) g^{\prime}(t)
$$

Proceeding as before, we obtain

$$
\begin{aligned}
& \left({ }_{g} F_{v, \alpha, \xi, y_{0}^{-}}^{\Psi, \gamma, \delta, k, \zeta} * g\right)(x ; p) \\
& \quad \leq E_{v, \alpha, \xi}^{\gamma, \delta, k, \zeta}\left(\eta\left(g\left(y_{0}\right)-g(x)\right)^{\nu} ; p\right) \Psi\left(g\left(y_{0}\right)-g(x)\right)\left|f^{\prime}\left(y_{0}\right)\right|
\end{aligned}
$$




$$
+G_{y_{0}}^{x}\left(E_{v, \alpha, \xi}^{\gamma, \delta, k, \zeta}, g ; \Psi\right) \varphi\left(\left|f^{\prime}(x)\right|,\left|f^{\prime}\left(y_{0}\right)\right|\right)\left(I\left(x, y_{0} ; g\right)-g(x)\right)
$$

and

$$
\begin{aligned}
\left({ }_{g} F_{v, \alpha, \xi, y}^{\Psi, \gamma, \delta, k}\right. & f * g)(x ; p) \\
\geq & -E_{v, \alpha, \xi}^{\gamma, \delta, k, \zeta}\left(\eta\left(g\left(y_{0}\right)-g(x)\right)^{v} ; p\right) \Psi\left(g\left(y_{0}\right)-g(x)\right)\left|f^{\prime}\left(y_{0}\right)\right| \\
\quad & \quad G_{y_{0}}^{x}\left(E_{v, \alpha, \xi}^{\gamma, \delta, k, \zeta}, g ; \Psi\right) \varphi\left(\left|f^{\prime}(x)\right|,\left|f^{\prime}\left(y_{0}\right)\right|\right)\left(I\left(x, y_{0} ; g\right)-g(x)\right) .
\end{aligned}
$$

We obtain inequality (2.22) by using (2.25), (2.26), (2.29), and (2.30).

Corollary 2 By setting $\mu=v$ in (2.22) we get the following inequality:

$$
\begin{aligned}
\mid\left({ }_{g} F_{\mu, \alpha, \xi, x_{0}^{+}}^{\Psi, \gamma, \delta, k, \zeta}\right. & f * g)(x ; p)+\left({ }_{g} F_{\mu, \alpha, \xi, y, y_{0}^{\prime}}^{\Psi, \gamma, k, \zeta} f * g\right)(x ; p) \mid \\
\leq & E_{\mu, \alpha, \xi, \xi}^{\gamma, \delta, \zeta}\left(\eta\left(g(x)-g\left(x_{0}\right)\right)^{\mu} ; p\right) \Psi\left(g(x)-g\left(x_{0}\right)\right)\left|f^{\prime}(x)\right| \\
& +G_{x}^{x_{0}}\left(E_{\mu, \alpha, \xi}^{\gamma, \delta, k, \zeta}, g ; \Psi\right) \varphi\left(\left|f^{\prime}\left(x_{0}\right)\right|,\left|f^{\prime}(x)\right|\right)\left(I\left(x_{0}, x ; g\right)-g(x)\right) \\
& +E_{\mu, \alpha, \xi}^{\gamma, \delta, k, \zeta}\left(\eta\left(g\left(y_{0}\right)-g(x)\right)^{\mu} ; p\right) \Psi\left(g\left(y_{0}\right)-g(x)\right)\left|f^{\prime}\left(y_{0}\right)\right| \\
& +G_{y_{0}}^{x}\left(E_{\mu, \alpha, \xi}^{\gamma, \delta, k, c}, g ; \Psi\right) \varphi\left(\left|f^{\prime}(x)\right|,\left|f^{\prime}\left(y_{0}\right)\right|\right)\left(I\left(x, y_{0} ; g\right)-g(x)\right) .
\end{aligned}
$$

Remark 4 For $\varphi(x, y)=x-y$ in (2.31), we get inequality (1.17) of Theorem 3 .

\section{Results for fractional and conformable integral operators}

In this section, we give bounds of some fractional and conformable fractional integral operators deduced from the results of Sect. 2.

Proposition 1 Under the assumptions of Theorem 4, we have

$$
\begin{aligned}
\Gamma(\alpha) & \left(\left({ }_{g}^{\alpha} I_{x_{0}^{+}} f\right)(x)+\left({ }_{g}^{\alpha} I_{y_{0}^{-}} f\right)(x)\right) \\
\leq & \left(g(x)-g\left(x_{0}\right)\right)^{\alpha} f(x)+\left(g\left(y_{0}\right)-g(x)\right)^{\alpha} f\left(y_{0}\right) \\
& +\left(g(x)-g\left(x_{0}\right)\right)^{\alpha-1} \varphi\left(f\left(x_{0}\right), f(x)\right)\left(I\left(x_{0}, x ; g\right)-g\left(x_{0}\right)\right) \\
& +\left(g\left(y_{0}\right)-g(x)\right)^{\alpha-1} \varphi\left(f(x), f\left(y_{0}\right)\right)\left(I\left(x, y_{0} ; g\right)-g(x)\right),
\end{aligned}
$$

where $\left({ }_{g}^{\alpha} I_{x_{0}^{+}} f\right)(x)$ and $\left({ }_{g}^{\alpha} I_{y_{0}^{-}} f\right)(x)$ are defined in [15].

Proof For $\Psi(t)=t^{\alpha}, \alpha>0$, and $p=\eta=0$ with $\mu=v$ in the proof of Theorem 4, bound (3.1) is satisfied.

For $\varphi(x, y)=x-y$ in (3.1), we get [16, Proposition 1].

Proposition 2 Under the assumptions of Theorem 4, we have

$$
\begin{aligned}
& \left(x_{0}^{+} I_{\Psi} f\right)(x)+\left(y_{0}^{-} I_{\Psi} f\right)(x) \\
& \quad \leq \Psi\left(x-x_{0}\right) f(x)+\frac{\Psi\left(x-x_{0}\right)}{2} \varphi\left(f\left(x_{0}\right), f(x)\right)+\Psi\left(y_{0}-x\right) f\left(y_{0}\right)
\end{aligned}
$$




$$
+\frac{\Psi\left(y_{0}-x\right)}{2} \varphi\left(f(x), f\left(y_{0}\right)\right)
$$

where $\left({ }_{x_{0}^{+}} I_{\Psi} f\right)(x)$ and $\left(y_{0}^{-} I_{\Psi} f\right)(x)$ are defined in [23].

Proof Using $g=I, \eta=p=0$, and $\mu=v$ in the proof of Theorem 4, bound (3.2) is satisfied.

For $\varphi(x, y)=x-y$ in (3.2), we get [16, Proposition 2]

Corollary 3 For $\Psi(t)=\frac{\Gamma(\alpha) t) \frac{\alpha}{k}}{k \Gamma_{k}(\alpha)}$ and $p=\eta=0,(1.12)$ and (1.13) reduce to fractional integrals (1.7) and (1.8). Further, the following bound for $\alpha \geq k$ is also satisfied:

$$
\begin{aligned}
&\left({ }_{g}^{\alpha} I_{x_{0}^{k}}^{k} f\right)(x)+\left({ }_{g}^{\alpha} I_{y_{0}^{-}}^{k} f\right)(x) \\
& \leq \frac{1}{k \Gamma_{k}(\alpha)}\left[\left(g(x)-g\left(x_{0}\right)\right)^{\frac{\alpha}{k}} f(x)+\left(g\left(y_{0}\right)-g(x)\right)^{\frac{\alpha}{k}} f\left(y_{0}\right)\right. \\
& \quad+\left(g(x)-g\left(x_{0}\right)\right)^{\frac{\alpha}{k}-1} \varphi\left(f\left(x_{0}\right), f(x)\right)\left(I\left(x_{0}, x ; g\right)-g\left(x_{0}\right)\right) \\
&\left.\quad+\left(g\left(y_{0}\right)-g(x)\right)^{\frac{\alpha}{k}-1} \varphi\left(f(x), f\left(y_{0}\right)\right)\left(I\left(x, y_{0} ; g\right)-g(x)\right)\right] .
\end{aligned}
$$

For $\varphi(x, y)=x-y$ in this inequality, we get [16, Corollary 12].

Corollary 4 Using $\Psi(t)=t^{\alpha}$ for $\alpha \geq 1$ and $g=I$ along with $p=\eta=0$, (1.12) and (1.13) give the fractional integral operators ${ }^{\alpha} I_{x_{0}} f(x)$ and ${ }^{\alpha} I_{y_{0}^{-}} f(x)$ defined in [15].

Moreover, the following bound is also satisfied:

$$
\begin{aligned}
& \Gamma(\alpha)\left(\left({ }^{\alpha} I_{x_{0}^{+}} f\right)(x)+\left({ }^{\alpha} I_{y_{0}^{-}} f\right)(x)\right) \\
& \quad \leq \frac{\left(x-x_{0}\right)^{\alpha}}{2}\left(2 f(x)+\varphi\left(f\left(x_{0}\right), f(x)\right)\right)+\frac{\left(y_{0}-x\right)^{\alpha}}{2}\left(2 f\left(y_{0}\right)+\varphi\left(f(x), f\left(y_{0}\right)\right)\right) .
\end{aligned}
$$

For $\varphi(x, y)=x-y$ in this inequality, we get [16, Corollary 13$]$.

Corollary 5 Using $\Psi(t)=\frac{\Gamma(\alpha) t \frac{\alpha}{k}}{k \Gamma_{k}(\alpha)}$ and $g=I$ along with $p=\eta=0$, (1.12) and (1.13) reduce to the following fractional integral operators given in [19]:

$$
\left(g F_{\mu, \alpha, \xi, x_{0}^{+}}^{\frac{\Gamma(\alpha) t)}{k \Gamma_{k}(\alpha)}, \gamma, \delta, k, \zeta} f\right)(x ; 0):={ }^{\alpha} I_{x_{0}^{+}}^{k} f(x), \quad\left({ }_{g} F_{\mu, \alpha, \xi, y, y_{0}^{-}}^{\frac{\Gamma(\alpha) t)}{k \Gamma_{k}(\alpha)}, \gamma, \delta, k, \zeta}, f\right)(x ; 0):={ }^{\alpha} I_{y_{0}^{-}}^{k} f(x)
$$

Moreover, the following bound is also satisfied for $\alpha \geq k$ :

$$
\begin{aligned}
& \left({ }^{\alpha} I_{x_{0}^{+}}^{k} f\right)(x)+\left({ }^{\alpha} I_{y_{0}}^{k} f\right)(x) \\
& \quad \leq \frac{1}{k \Gamma_{k}(\alpha)}\left[\frac{\left(x-x_{0}\right)^{\frac{\alpha}{k}}}{2}\left(2 f(x)+\varphi\left(f\left(x_{0}\right), f(x)\right)\right)+\frac{\left(y_{0}-x\right)^{\frac{\alpha}{k}}}{2}\left(2 f\left(y_{0}\right)+\varphi\left(f(x), f\left(y_{0}\right)\right)\right)\right] .
\end{aligned}
$$

For $\varphi(x, y)=x-y$ in this inequality, we get [16, Corollary 14]. 
Corollary 6 Using $\Psi(t)=t^{\alpha}, \alpha>0$, and $g(x)=\frac{x^{\rho}}{\rho}, \rho>0$, in (1.12) and (1.13), respectively, with $p=\eta=0$, we obtain the following fractional integral operators given in [4]:

$$
\begin{aligned}
& \left({ }_{g} F_{\mu, \alpha, \xi, x_{0}^{+}}^{t^{\alpha}, \gamma, k, \zeta} f\right)(x ; 0)=\left({ }^{\rho} I_{x_{0}^{+}}^{\alpha} f\right)(x)=\frac{\rho^{1-\alpha}}{\Gamma(\alpha)} \int_{x_{0}}^{x}\left(x^{\rho}-t^{\rho}\right)^{\alpha-1} t^{\rho-1} f(t) d t, \\
& \left({ }_{g} F_{\mu, \alpha, \xi, y_{0}^{\prime}}^{t^{\alpha}, \gamma, k, \zeta} f\right)(x ; 0)=\left({ }^{\rho} I_{y_{0}}^{\alpha} f\right)(x)=\frac{\rho^{1-\alpha}}{\Gamma(\alpha)} \int_{x}^{y_{0}}\left(t^{\rho}-x^{\rho}\right)^{\alpha-1} t^{\rho-1} f(t) d t .
\end{aligned}
$$

Moreover, the following bound is also satisfied:

$$
\begin{aligned}
& \left({ }^{\rho} I_{x_{0}^{+}}^{\alpha} f\right)(x)+\left({ }^{\rho} I_{y_{0}}^{\alpha} f\right)(x) \\
& \quad \leq \frac{1}{\rho^{\alpha} \Gamma(\alpha)}\left[\left(x^{\rho}-x_{0}^{\rho}\right)^{\alpha} f(x)+\left(y_{0}^{\rho}-x^{\rho}\right)^{\alpha} f\left(y_{0}\right)+\left(x^{\rho}-x_{0}^{\rho}\right)^{\alpha-1} \varphi\left(f\left(x_{0}\right), f(x)\right)\right. \\
& \left.\quad \times\left(\frac{x^{\rho+1}-x_{0}^{\rho+1}}{\left(x-x_{0}\right)(\rho+1)}-x_{0}^{\rho}\right)+\left(y_{0}^{\rho}-x^{\rho}\right)^{\alpha-1} \varphi\left(f(x), f\left(y_{0}\right)\right)\left(\frac{y_{0}^{\rho+1}-x^{\rho+1}}{\left(y_{0}-x\right)(\rho+1)}-x^{\rho}\right)\right] .
\end{aligned}
$$

For $\varphi(x, y)=x-y$ in this inequality, we get [16, Corollary 15].

Corollary 7 Using $\Psi(t)=t^{\alpha}, \alpha>0$, and $g(x)=\frac{x^{s+1}}{s+1}, s>0$, in (1.12) and (1.13), respectively, with $p=\eta=0$, we obtain the following fractional integral operators:

$$
\begin{aligned}
& \left({ }_{g} t_{\mu, \alpha, \xi, x_{0}^{+}}^{t^{\alpha}, \gamma, \delta, k, \zeta} f\right)(x ; 0) \\
& \quad=\left({ }^{s} I_{x_{0}^{+}}^{\alpha} f\right)(x)=\frac{(s+1)^{1-\alpha}}{\Gamma(\alpha)} \int_{x_{0}}^{x}\left(x^{s+1}-t^{s+1}\right)^{\alpha-1} t^{s} f(t) d t, \\
& \left(F_{g}^{t_{\mu, \alpha, \xi, y_{0}^{-}}^{\alpha}, \gamma, \delta, k, \zeta}\right)(x ; 0) \\
& \quad=\left({ }^{s} I_{y_{0}^{\alpha}}^{\alpha} f\right)(x)=\frac{(s+1)^{1-\alpha}}{\Gamma(\alpha)} \int_{x}^{y_{0}}\left(t^{s+1}-x^{s+1}\right)^{\alpha-1} t^{s} f(t) d t .
\end{aligned}
$$

Moreover, the following bound is also satisfied:

$$
\begin{aligned}
&\left({ }^{s} I_{x_{0}^{\alpha}}^{\alpha} f\right)(x)+\left({ }^{s} I_{y_{0}^{-}}^{\alpha} f\right)(x) \\
& \leq \frac{1}{(s+1)^{\alpha} \Gamma(\alpha)}\left[\left(x^{s+1}-x_{0}^{s+1}\right)^{\alpha} f(x)+\left(x^{s+1}-x_{0}^{s+1}\right)^{\alpha-1} \varphi\left(f\left(x_{0}\right), f(x)\right)\right. \\
& \quad \times\left(\frac{x^{s+2}-x_{0}^{s+2}}{\left(x-x_{0}\right)(s+2)}-x_{0}^{s+1}\right)+\left(y_{0}^{s+1}-x^{s+1}\right)^{\alpha} f\left(y_{0}\right)+\left(y_{0}^{s+1}-x^{s+1}\right)^{\alpha-1} \varphi\left(f(x), f\left(y_{0}\right)\right) \\
&\left.\quad \times\left(\frac{y_{0}^{s+2}-x^{s+2}}{\left(y_{0}-x\right)(s+2)}-x^{s+1}\right)\right] .
\end{aligned}
$$

For $\varphi(x, y)=x-y$ in this inequality, we get [16, Corollary 16$]$.

Corollary 8 Using $\Psi(t)=\frac{\Gamma(\alpha) t^{\frac{\alpha}{k}}}{k \Gamma_{k}(\alpha)}$ and $g(x)=\frac{x^{s+1}}{s+1}, s>0$, in (1.12) and (1.13), respectively, with $p=\eta=0$, we obtain the following fractional integral operators given in [22]:

$$
\left({ }_{g} F_{\mu, \alpha, \xi, x_{0}^{+}}^{\frac{\Gamma(\alpha) k^{\frac{\alpha}{k}}}{k \Gamma_{k}(\alpha, \gamma, \delta, k, \zeta}} f\right)(x ; 0)
$$




$$
\begin{aligned}
& =\left({ }_{k}^{s} I_{x_{0}^{\alpha}}^{\alpha} f\right)(x)=\frac{(s+1)^{1-\frac{\alpha}{k}}}{k \Gamma_{k}(\alpha)} \int_{x_{0}}^{x}\left(x^{s+1}-t^{s+1}\right)^{\frac{\alpha}{k}-1} t^{s} f(t) d t, \\
& \frac{\Gamma(\alpha) t}{\frac{\alpha}{k}}, \gamma, \delta, k, \zeta \\
& \left(F_{g, \alpha, \xi, y_{0}^{-}}^{\frac{\Gamma(\alpha)}{k \Gamma_{k}(\alpha)}, \gamma, \delta, k, \zeta} f\right)(x ; 0) \\
& =\left({ }_{k}^{s} I_{y_{0}^{-}}^{\alpha} f\right)(x)=\frac{(s+1)^{1-\frac{\alpha}{k}}}{k \Gamma_{k}(\alpha)} \int_{x}^{y_{0}}\left(t^{s+1}-x^{s+1}\right)^{\frac{\alpha}{k}-1} t^{s} f(t) d t .
\end{aligned}
$$

Moreover, the following bound is also satisfied:

$$
\begin{aligned}
\left({ }_{k}^{s} I_{x_{0}^{+}}^{\alpha} f\right)(x)+\left({ }_{k}^{s} I_{y_{0}^{-}}^{\alpha} f\right)(x) \\
\leq \frac{1}{(s+1)^{\frac{\alpha}{k}} k \Gamma_{k}(\alpha)}\left[\left(x^{s+1}-x_{0}^{s+1}\right)^{\frac{\alpha}{k}} f(x)+\left(x^{s+1}-x_{0}^{s+1}\right)^{\frac{\alpha}{k}-1} \varphi\left(f\left(x_{0}\right), f(x)\right)\right. \\
\quad \times\left(\frac{x^{s+2}-x_{0}^{s+2}}{\left(x-x_{0}\right)(s+2)}-x_{0}^{s+1}\right)+\left(y_{0}^{s+1}-x^{s+1}\right)^{\frac{\alpha}{k}} f\left(y_{0}\right)+\left(y_{0}^{s+1}-x^{s+1}\right)^{\frac{\alpha}{k}-1} \varphi\left(f(x), f\left(y_{0}\right)\right) \\
\left.\quad \times\left(\frac{y_{0}^{s+2}-x^{s+2}}{\left(y_{0}-x\right)(s+2)}-x^{s+1}\right)\right] .
\end{aligned}
$$

For $\varphi(x, y)=x-y$ in this inequality, we get [16, Corollary 17$]$.

Corollary 9 Using $\Psi(t)=t^{\alpha}, \alpha>0$, and $g(x)=\frac{x^{\beta+s}}{\beta+s}, \beta, s>0$, in (1.12) and (1.13), respectively, with $p=\eta=0$, we obtain the following fractional integral operators given in [12]:

$$
\begin{aligned}
& \left({ }_{g} F_{\mu, \alpha, \xi, x_{0}^{+}}^{t^{\alpha}, \gamma, k} f\right)(x ; 0) \\
& \quad=\left({ }_{\beta}^{s} I_{x_{0}^{+}}^{\alpha} f\right)(x)=\frac{(\beta+s)^{1-\alpha}}{\Gamma(\alpha)} \int_{x_{0}}^{x}\left(x^{\beta+s}-t^{\beta+s}\right)^{\alpha-1} t^{s} f(t) d t, \\
& \left({ }_{g} F_{\mu, \alpha, \xi, y_{0}^{\prime}}^{t^{\alpha}, \gamma, \delta} f\right)(x ; 0) \\
& \quad=\left({ }_{\beta}^{s} I_{y_{0}^{-}}^{\alpha} f\right)(x)=\frac{(\beta+s)^{1-\alpha}}{\Gamma(\alpha)} \int_{x}^{y_{0}}\left(t^{\beta+s}-x^{\beta+s}\right)^{\alpha-1} t^{s} f(t) d t .
\end{aligned}
$$

Moreover, the following bound is also satisfied:

$$
\begin{aligned}
&\left({ }_{\beta}^{s} I_{x_{0}^{\alpha}}^{\alpha} f\right)(x)+\left({ }_{\beta}^{s} I_{y_{0}}^{\alpha} f\right)(x) \\
& \leq \frac{1}{(\beta+s)^{\alpha} \Gamma(\alpha)}\left[\left(x^{\beta+s}-x_{0}^{\beta+s}\right)^{\alpha} f(x)+\left(x^{\beta+s}-x_{0}^{\beta+s}\right)^{\alpha-1} \varphi\left(f\left(x_{0}\right), f(x)\right)\right. \\
& \times\left(\frac{x^{\beta+s+1}-x_{0}^{\beta+s+1}}{\left(x-x_{0}\right)(\beta+s+1)}-x_{0}^{\beta+s}\right)+\left(y_{0}^{\beta+s}-x^{\beta+s}\right)^{\alpha-1} \varphi\left(f(x), f\left(y_{0}\right)\right) \\
&\left.\times\left(\frac{y_{0}^{\beta+s+1}-x^{\beta+s+1}}{\left(y_{0}-x\right)(\beta+s+1)}-x^{\beta+s}\right)+\left(y_{0}^{\beta+s}-x^{\beta+s}\right)^{\alpha} f\left(y_{0}\right)\right] .
\end{aligned}
$$

For $\varphi(x, y)=x-y$ in this inequality, we get [16, Corollary 18$]$.

Corollary 10 Using $\Psi(t)=t^{\alpha}, \alpha>0$, and $g(x)=\frac{\left(x-x_{0}\right)^{\rho}}{\rho}$ in (1.12) and $g(x)=\frac{-\left(y_{0}-x\right)^{\rho}}{\rho}$ in (1.13), where $\rho>0$ with $p=\eta=0$, we obtain the following fractional integral operators 
given in [10]:

$$
\begin{aligned}
& \left({ }_{g} F_{\mu, \alpha, \xi, x_{0}^{+}}^{t^{\alpha}, \gamma, \delta, \zeta} f\right)(x ; 0) \\
& \quad=\left({ }^{\rho} I_{x_{0}^{+}}^{\alpha} f\right)(x)=\frac{\rho^{1-\alpha}}{\Gamma(\alpha)} \int_{x_{0}}^{x}\left(\left(x-x_{0}\right)^{\rho}-\left(t-x_{0}\right)^{\rho}\right)^{\alpha-1}\left(t-x_{0}\right)^{\rho-1} f(t) d t, \\
& \left({ }_{g} F_{\mu, \alpha, \xi, \xi, y}^{t^{\alpha}, \gamma, \delta, k, \zeta} f\right)(x ; 0) \\
& \quad=\left({ }^{\rho} I_{y_{0}}^{\alpha} f\right)(x)=\frac{\rho^{1-\alpha}}{\Gamma(\alpha)} \int_{x}^{y_{0}}\left(\left(y_{0}-x\right)^{\rho}-\left(y_{0}-t\right)^{\rho}\right)^{\alpha-1}\left(y_{0}-t\right)^{\rho-1} f(t) d t .
\end{aligned}
$$

Moreover, the following bound is also satisfied:

$$
\begin{aligned}
\left({ }^{\rho} I_{x_{0}^{\alpha}}^{\alpha} f\right)(x)+\left({ }^{\rho} I_{y_{0}}^{\alpha} f\right)(x) & \\
\leq & \frac{1}{\rho^{\alpha} \Gamma(\alpha)}\left[\left(x-x_{0}\right)^{\rho \alpha} f(x)+\varphi\left(f\left(x_{0}\right), f(x)\right) \frac{\left(x-x_{0}\right)^{\rho \alpha}}{\rho+1}\right. \\
& \left.+\left(y_{0}-x\right)^{\rho \alpha} f\left(y_{0}\right)+\varphi\left(f(x), f\left(y_{0}\right)\right) \frac{\rho\left(y_{0}-x\right)^{\rho \alpha}}{\rho+1}\right] .
\end{aligned}
$$

For $\varphi(x, y)=x-y$ in this inequality, we get [16, Corollary 19$]$.

Corollary 11 For $\Psi(t)=\frac{\Gamma(\alpha) \frac{\alpha}{k}}{k \Gamma_{k}(\alpha)}, \alpha>k$, and $g(x)=\frac{\left(x-x_{0}\right)^{\rho}}{\rho}$ in $(1.12)$ and $g(x)=\frac{-\left(y_{0}-x\right)^{\rho}}{\rho}$ in (1.13), where $\rho>0$ with $p=\eta=0$, we obtain the following fractional integral operators given in $[8]$ :

$$
\begin{aligned}
& \left(g_{\mu, \alpha, \xi, x_{0}^{+}}^{\frac{\Gamma(\alpha) t)}{k \Gamma_{k}(\alpha)}, \gamma, \delta, k, \zeta} f\right)(x ; 0) \\
& =\left({ }_{k}^{\rho} I_{x_{0}^{\alpha}}^{\alpha} f\right)(x)=\frac{\rho^{1-\frac{\alpha}{k}}}{k \Gamma_{k}(\alpha)} \int_{x_{0}}^{x}\left(\left(x-x_{0}\right)^{\rho}-\left(t-x_{0}\right)^{\rho}\right)^{\frac{\alpha}{k}-1}\left(t-x_{0}\right)^{\rho-1} f(t) d t,
\end{aligned}
$$

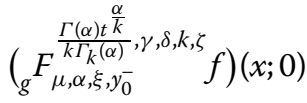

$$
\begin{aligned}
& =\left({ }_{k}^{\rho} I_{y_{0}^{\alpha}}^{\alpha} f\right)(x)=\frac{\rho^{1-\frac{\alpha}{k}}}{k \Gamma_{k}(\alpha)} \int_{x}^{y_{0}}\left(\left(y_{0}-x\right)^{\rho}-\left(y_{0}-t\right)^{\rho}\right)^{\frac{\alpha}{k}-1}\left(y_{0}-t\right)^{\rho-1} f(t) d t .
\end{aligned}
$$

Moreover, the following bound is also satisfied:

$$
\begin{aligned}
& \left({ }_{k}^{\rho} I_{x_{0}^{+}}^{\alpha} f\right)(x)+\left({ }_{k}^{\rho} I_{y_{0}}^{\alpha} f\right)(x) \\
& \leq \frac{1}{\rho^{\frac{\alpha}{k}} k \Gamma_{k}(\alpha)}\left[\left(x-x_{0}\right)^{\frac{\rho \alpha}{k}} f(x)+\varphi\left(f\left(x_{0}\right), f(x)\right) \frac{\left(x-x_{0}\right)^{\frac{\rho \alpha}{k}}}{\rho+1}\right. \\
& \left.\quad+\left(y_{0}-x\right)^{\frac{\rho \alpha}{k}} f\left(y_{0}\right)+\varphi\left(f(x), f\left(y_{0}\right)\right) \frac{\rho\left(y_{0}-x\right)^{\frac{\rho \alpha}{k}}}{\rho+1}\right] .
\end{aligned}
$$

For $\varphi(x, y)=x-y$ in this inequality, we get [16, Corollary 20].

By applying Theorems 5 and 6 we can obtain results for fractional and conformable fractional integral operators associated with the unified integral operators, which we leave for the reader. 


\section{Concluding remarks}

In this paper, we study the unified integral operators (1.12) and (1.13) for the notion of $\varphi$-convex functions. For $\varphi$-convex functions, we investigated bounds of these operators in different forms, which lead to bounds of several known fractional and conformable fractional integral operators. We identified some results for fractional integral operators in Sect. 3. Also, we identified connections with the known results.

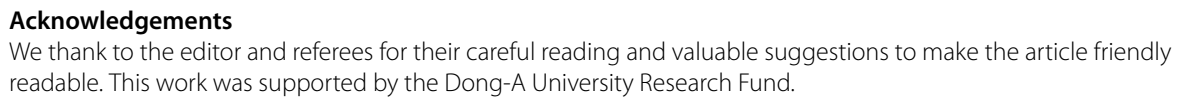

Funding

There is no funding available for the publication of this paper.

Availability of data and materials

There is no additional data required for the finding of results of this paper.

Competing interests

The authors declare that they have no competing interests.

Authors' contributions

All authors have equal contribution in this paper. All authors read and approved the final manuscript.

\section{Author details}

'Department of Mathematics, Dong-A University, Busan, South Korea. ²Department of Mathematics, University of Wah, Wah Cantt, Pakistan. ${ }^{3}$ Department of Mathematics, COMSATS University Islamabad, Attock, Pakistan. ${ }^{4}$ Department of Mathematics, Gyeongsang National University, Jinju, South Korea.

\section{Publisher's Note}

Springer Nature remains neutral with regard to jurisdictional claims in published maps and institutional affiliations.

Received: 16 March 2020 Accepted: 7 June 2020 Published online: 16 June 2020

\section{References}

1. Anastassiou, G.A.: Generalized fractional Hermite-Hadamard inequalities involving $m$-convexity and $(s, m)$-convexity. Ser. Math. Inform. 28(2), 107-126 (2013)

2. Andrić, M., Farid, G., Pečarić, J.: A further extension of Mittag-Leffler function. Fract. Calc. Appl. Anal. 21(5), 1377-1395 (2018)

3. Bombardelli, M., Varošanec, S.: Properties of $h$-convex functions related to the Hermite-Hadamard-Fejér inequalities. Comput. Math. Appl. 58(9), 1869-1877 (2009)

4. Chen, H., Katugampola, U.N.: Hermite-Hadamard and Hermite-Hadamard-Fejér type inequalities for generalized fractional integrals. J. Math. Anal. Appl. 446, 1274-1291 (2017)

5. Du, T., Wang, H., Khan, M.A., Zhang, Y.: Certain integral inequalities considering generalized $m$-convexity on fractal sets and their applications. Fractals 27(7), 1-17 (2019)

6. Farid, G.: A unified integral operator and further its consequences. Open J. Math. Anal. 4(1), 1-7 (2020)

7. Gordji, M.E., Delavar, M.R., De la Sen, M.: On $\varphi$-convex functions. J. Math. Inequal. 10(1), 173-183 (2016)

8. Habib, S., Mubeen, S., Naeem, M.N.: Chebyshev type integral inequalities for generalized $k$-fractional conformable integrals. J. Inequal. Spec. Funct. 9(4), 53-65 (2018)

9. Hudzik, H., Maligranda, L:: Some remarks on s-convex functions. Aequ. Math. 48, 100-111 (1994)

10. Jarad, F., Ugurlu, E., Abdeljawad, T., Baleanu, D.: On a new class of fractional operators. Adv. Differ. Equ. 2017, 247 (2017)

11. Khan, M.A., Mohammad, N., Nwaeze, E.R., Chu, Y.-M.: Quantum Hermite-Hadamard inequality by means of a Green function. Adv. Differ. Equ. 2020, 99 (2020)

12. Khan, T.U., Khan, M.A.: Generalized conformable fractional operators. J. Comput. Appl. Math. 346, 378-389 (2019)

13. Khurshid, Y., Khan, M.A., Chu, Y.-M.: Conformable integral inequalities of the Hermite-Hadamard type in terms of GG and GA-convexities. J. Funct. Spaces 2019, Article ID 6926107 (2019)

14. Khurshid, Y., Khan, M.A., Chu, Y.-M., Khan, Z.A.: Hermite-Hadamard-Fejér inequalities for conformable fractional integrals via preinvex functions. J. Funct. Spaces 2019, Article ID 3146210 (2019)

15. Kilbas, A.A., Srivastava, H.M., Trujillo, J.J.: Theory and Applications of Fractional Differential Equations. North-Holland Mathematics Studies, vol. 204. Elsevier, New York (2006)

16. Kwun, Y.C., Farid, G., Nazeer, W., Ullah, S., Mahreen, K., Kang, S.M.: Inequalities for a unified integral operator and associated results in fractional integrals. IEEE Access 7, 126283-126292 (2019)

17. Mehmood, S., Farid, G., Khan, K.A., Yussouf, M.: New Hadamard and Fejér-Hadamard fractional inequalities for exponentially m-convex function. Eng. Appl. Sci. Lett. 3(1), 45-55 (2020)

18. Mehmood, S., Farid, G., Khan, K.A., Yussouf, M.: New fractional Hadamard and Fejér-Hadamard inequalities associated with exponentially $(h, m)$-convex function. Eng. Appl. Sci. Lett. 3(2), 9-18 (2020) 
19. Mubeen, S., Habibullah, G.M.: k-Fractional integrals and applications. Int. J. Contemp. Math. Sci. 7, 89-94 (2012)

20. Özdemir, M.E., Akdemri, A.O., Set, E.: On (h, m)-convexity and Hadamard type inequalities. Transylv. J. Math. Mech. 8(1), $51-58(2016)$

21. Roberts, A.W., Varberg, D.E.: Convex Functions. Academic Press, New York (1973)

22. Sarikaya, M.Z., Dahmani, M., Kiris, M.E., Ahmad, F.: (k, s)-Riemann-Liouville fractional integral and applications. Hacet. J. Math. Stat. 45(1), 77-89 (2016). https://doi.org/10.15672/HJMS.20164512484

23. Sarikaya, M.Z., Ertuğral, F.: On the generalized Hermite-Hadamard inequalities https://www.researchgate.net/publication/321760443 (2017). [Online]

24. Yuan, S.M., Liu, Z.M.: Some properties of $\alpha$-convex and $\alpha$-quasiconvex functions with respect to $n$-symmetric points. Appl. Math. Comput. 188(2), 1142-1150 (2007)

\section{Submit your manuscript to a SpringerOpen ${ }^{\circ}$} journal and benefit from:

- Convenient online submission

- Rigorous peer review

- Open access: articles freely available online

- High visibility within the field

- Retaining the copyright to your article

Submit your next manuscript at $\gg$ springeropen.com 